\title{
Formation of a sonic horizon in isotropically expanding Bose-Einstein condensates
}

\author{
Yasunari Kurita \\ Osaka City University Advanced Mathematical Institute, Osaka 558-8585, Japan \\ Takao Morinari \\ Yukawa Institute for Theoretical Physics, Kyoto University, Kyoto 606-8502, Japan
}

\begin{abstract}
We propose a simple experiment to create a sonic horizon in isotropically trapped cold atoms within currently available experimental techniques. Numerical simulation of the Gross-Pitaevskii equation shows that the sonic horizon should appear by making the condensate expand. The expansion is triggered by changing the interaction which can be controlled by the Feshbach resonance in real experiments. The sonic horizon is shown to be quasi-static for sufficiently strong interaction or large number of atoms. The characteristic temperature that is associated with particle emission from the horizon, which corresponds to the Hawking temperature in an ideal situation, is estimated to be a few nK.

PACS numbers: 03.75.Kk, 03.75.Hh, 04.62.+v, 05.30.Jp
\end{abstract}

\section{INTRODUCTION}

For the exploration of cosmology and gravitational physics, it is necessary to have a deep understanding of quantum filed theory in curved spacetime: It is widely believed that everything except for the spacetime itself should originate from quantum fluctuations in the early Universe. Quantum effects on curved spacetime, such as the Hawking radiation, give us theoretical support for black hole thermodynamics. However, it is extremely hard to verify such quantum effects experimentally. For instance, the Hawking radiation is thermal radiation emitted from a dynamically formed stationary black hole [1]. However, the characteristic temperature of the thermal radiation, the Hawking temperature, is on the order of several tens of nanokelvins at most, which is much lower than the cosmic microwave background radiation temperature. So detecting thermal radiation from a real black hole is almost impossible.

One way to circumvent this difficulty is to make use of artificial black holes 2] 3]. Unruh showed in his seminal paper [4] that excitations in a supersonic flow corresponds to a scalar field equation on a curved spacetime including a horizon. Since the phenomenon of the Hawking radiation can be separated from gravitational physics, it is possible to detect the corresponding phenomenon in a fluid system with sonic horizon [4]. The basic idea is to identify fluid flow with curved spacetime and excitation modes with fields on the curved spacetime. A black hole event horizon corresponds to a sonic horizon in a fluid. For the purpose of investigating the quantum effects, a quantum fluid should be considered. As such a quantum fluid, Bose-Einstein condensates (BEC) in trapped cold atoms [5, [6] are one of the most suitable systems [7]-[9]. A crucial advantage is that one can control scattering length between atoms by making use of the Feshbach resonance[10]. In fact, that experimental technique was used in observing jets and bursts in a collapsing condensate, which is called "Bose-Novae" [1]]. An remarkable explanation of burst and jet phenomena in Bose-Novae was proposed in 12 13, based on quantum field theory of particle creation and structure formation in cosmological spacetime.

In order to verify the Hawking effect in fluid analogy, it is necessary to create a stationary sonic horizon because it is a phenomenon on a dynamically formed stationary black hole. Although several possibilities have been discussed so far [7, 8], it seems difficult to realize exactly stationary sonic horizon in cold atoms. However, if one can make a quasi-static horizon for high frequency modes, particle emission from the horizon is also expected. In this paper, we numerically demonstrate that a quasistatic horizon is realized without introducing new experimental techniques beyond currently available ones. We consider an expanding BEC driven by a sudden change of the interaction. Numerically solving the Gross-Pitaevskii (GP) equation, we show that a quasi-static horizon appear.

We note that there have been made great efforts to create cosmological geometry using expanding BEC [14]- 21] . In these papers, the analogue models with specific cosmological metrics such as Friedmann-Robertson-Walker (FRW) metric or de Sitter metric were discussed and the effects of particle creation in these cosmological spacetimes were investigated. However, in this paper, we do not intend to obtain any cosmological analogue model with well-known analytic metric. But we try to obtain dynamically formed quasi-static sonic horizon. Furthermore, the sonic horizon should be formed in hydrodynamic regime of the condensate because the spacetime analogy is only valid in such regime. The appearance of a horizon due to expansion of a condensate was noticed in the previous works, for example in [15], and its formation itself is not surprising. But it is non-trivial whether the condensate flow at the horizon is in the hydrodynamic regime, or not. In this paper, we show that the quasi-static sonic horizon will appear in the hydrodynamic regime of the condensate by changing the atomic interaction instantaneously. 


\section{ANALOGUE SPACETIME IN BEC}

In the coherent state path integral formulation, the action of bosons is given by

$$
\begin{array}{r}
S=\int d t \int d^{3} \mathbf{r}\left(i \hbar \bar{\phi} \partial_{t} \phi-\frac{\hbar^{2}}{2 m} \nabla \bar{\phi} \cdot \nabla \phi-V_{\text {ext }} \bar{\phi} \phi\right. \\
\left.-\frac{1}{2} U_{0}(\bar{\phi} \phi)^{2}\right)
\end{array}
$$

where $V_{\text {ext }}$ is the confining potential and $U_{0}=4 \pi \hbar^{2} a / m$ with $a$ the $s$-wave scattering length. For $\phi$ and $V_{\text {ext }}$, spatial and time dependences are implicit. The saddle point equation for this action leads to the GP equation:

$$
i \hbar \partial_{t} \Psi=\left(-\frac{\hbar^{2}}{2 m} \nabla^{2}+V_{\text {ext }}+U_{0}|\Psi|^{2}\right) \Psi .
$$

This GP equation governs the dynamics of the condensate whose order parameter is given by $\Psi$.

Now we consider hydrodynamical approximation. We denote the bosonic field $\phi$ as $\phi=\sqrt{\rho_{0}+\rho} e^{i\left(\varphi_{0}+\varphi\right)}$, where $\sqrt{\rho_{0}}$ and $\varphi_{0}$ are the amplitude and the phase of $\Psi$, respectively. (Namely, $\Psi=\sqrt{\rho_{0}} \exp \left(i \varphi_{0}\right)$.) The fields $\rho$ and $\varphi$ describe the non-condensate part of the bosonic field. If the density gradient is sufficiently smooth over the scale determined by the local healing length $\xi(\mathbf{r}, t) \equiv$ $\hbar /\left(2 m \rho_{0} U_{0}\right)^{1 / 2}$, or, in other words if the conditions,

$$
\left|\xi \nabla \rho_{0} / \rho_{0}\right|^{2} \ll 1 \quad \text { and } \quad|\xi \nabla \rho / \rho|^{2} \ll 1,
$$

are satisfied, hydrodynamical approximation is justified. (The condition (3) shall be examined later.) Under the above condition, the equation for $\rho$ is

$$
\rho=-\hbar\left(\dot{\varphi}+\mathbf{v}_{\mathbf{0}} \cdot \nabla \varphi\right) / U_{0},
$$

where $\mathbf{v}_{\mathbf{0}}=\frac{\hbar}{m} \nabla \varphi_{0}$ is the background fluid velocity, and the effective action for $\varphi$ is

$$
S_{\mathrm{eff}}=\int d t \int d^{3} \mathbf{r} \frac{\hbar^{2}}{2 U_{0}}\left[\left(\dot{\varphi}+\mathbf{v}_{\mathbf{0}} \cdot \nabla \varphi\right)^{2}-\frac{\rho_{0} U_{0}}{m}(\nabla \varphi)^{2}\right]
$$

Taking variation with respect to $\varphi$, we find that the field equation for $\varphi$ has the form of a propagating wave equation. Also, the equation for the field $\varphi$ can be expressed as $\partial_{\mu}\left(\sqrt{-g} g^{\mu \nu} \partial_{\nu}\right) \varphi=0$, where $g^{\mu \nu}$ is the inverse of the following matrix:

$$
g_{\mu \nu} \propto\left(\begin{array}{cc}
-\left(c_{s}^{2}-\mathbf{v}_{\mathbf{0}}^{2}\right) & -\mathbf{v}_{\mathbf{0}} \\
-\mathbf{v}_{\mathbf{0}} & \mathbf{1}
\end{array}\right),
$$

with $c_{s}=\sqrt{\rho_{0} U_{0} / m}$ and $g=\operatorname{det} g_{\mu \nu}$. Thus, the equation is equivalent to an equation for a massless field on a curved spacetime determined by the metric (6) with $c_{s}$ the speed of "light." Note that in order to interpret the quantity $c_{s}$ as a velocity, $U_{0}$ must be positive because, for negative $U_{0}, c_{s}$ becomes pure imaginary. Hereafter we consider positive $U_{0}$, which leads to an effective spacetime with Lorentzian signature.
For the excitation modes of $\varphi$ whose frequencies, say $\omega$, are much higher than the frequency $\omega_{\mathrm{BEC}}$, which is associated with the condensate motion, the condensate will be quasi-static. (For moderate changes of the interaction, $\omega_{\mathrm{BEC}}$ turns out to be the trapping harmonic potential frequency $\omega_{h o}$, as shall be discussed below .) The analogy between fields on the curved spacetime and excitation modes on the fluid flow is meaningful only when the conditions (3) are satisfied. The latter condition in Eq.(3) turns out to be $\omega^{2} \ll\left(c_{s} / \xi\right)^{2}$, by using Eq. (4). Thus, the frequency $\omega$ has an upper limit. The former condition in Eq. (3) is satisfied in the regions far from the edge of the condensate. (In contrast, if one is very close to the edge, zero-point oscillations become dominant, and so the former condition in (3) is not satisfied.) If there exists intermediate region for $\omega$ of

$$
\omega_{\mathrm{BEC}} \ll \omega \ll c_{s} / \xi,
$$

then the hydrodynamical approximation is justified and the condensate is quasi-static for excitation modes. Note that those modes are associated with particle emission from the horizon if the hydrodynamical flow has a dynamically formed sonic horizon. The necessary condition for the existence of the intermediate region (7) is

$$
\frac{c_{s}}{\xi \omega_{\mathrm{BEC}}} \gg 1
$$

In the following, we mainly consider condensate satisfying the above condition.

\section{FORMATION OF SONIC HORIZON}

Now we investigate sonic horizon formation in an expanding BEC trapped in isotropic harmonic potential, $V_{\text {ext }}=m \omega_{h o}^{2} r^{2} / 2$, where $r$ is the radial coordinate. Initially, we set the condensate in a ground state with an initial atomic interaction $a_{i}$. At $t=0$, the atomic interaction is changed suddenly from $a_{i}$ to $a_{f}\left(>a_{i}\right)$, which makes the condensate expand. Then, formation of sonic horizon can be expected. The reason is as follows: The sound velocity is proportional to square root of the condensate density and a decreasing function of $r$. In contrast, the fluid velocity is an increasing function of $r$ and the condensate expands fast around its edge whereas $v_{0}(\mathbf{r}=\mathbf{0})=0$ due to the boundary condition. Therefore, at an intermediate radius, $v_{0}$ exceeds $c_{s}$ and the fluid flow is transonic. It has a surface satisfying $c_{s}=\left|v_{0}\right|$ which is called a sonic horizon. We should note that the sonic horizon corresponds to a horizon in the analogue spacetime defined by the metric (6) . We also note that the subsonic region is around the center of the condensate and inside of the sonic horizon.

In general, if a fluid has a static sonic horizon and a proper quantum state for an excitation field is realized, then it is theoretically predicted that the horizon will emit thermal radiation of the quantum field. As will 


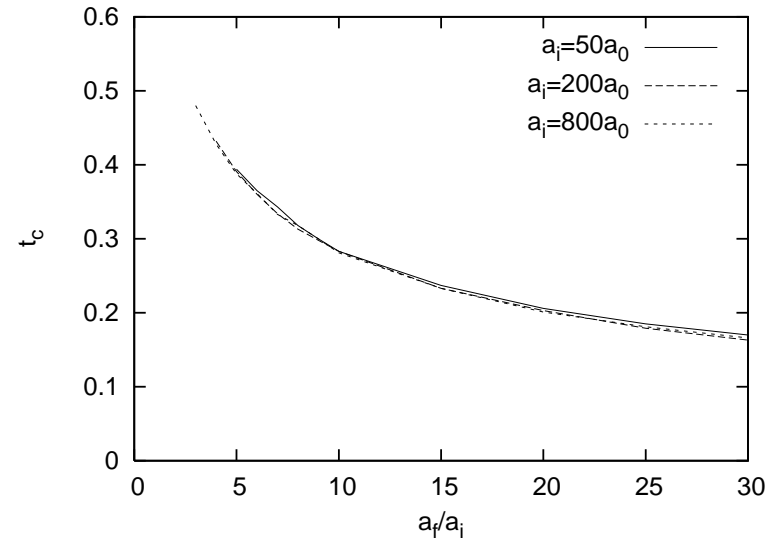

FIG. 1: Times $t_{c}$ in each simulation are shown in units of $\omega_{h o}^{-1}$. The horizontal axis is the ratio of $a_{f}$ to $a_{i}$.

be discussed in Appendix $\mathrm{A}$, if the sonic horizon in the expanding condensate is quasi-static for the field $\varphi$, the horizon will emit thermal radiation into the center of the condensate. The temperature characterizing the thermal emission (Hawking temperature) is given by the following formula:

$$
T_{\mathrm{pc}}=\left.\frac{\hbar}{2 \pi k_{\mathrm{B}}} \partial_{r}\left(v_{0}-c_{s}\right)\right|_{r_{\mathrm{H}}},
$$

where $r_{\mathrm{H}}$ is the horizon radius and $k_{\mathrm{B}}$ is the Boltzmann's constant [4] 24]. From the above expression, it is found that the Hawking temperature is determined by gradient of fluid and sound velocity at the horizon. Thus, it is important to investigate the velocity gradients at the horizon. In the derivation of the formula (9), it is assumed that the dynamically formed horizon is static, but in actual experiments, this assumption is not satisfied exactly. Therefore, the spectrum of the particle is not fully given by the single Planck's distribution function, but rather given by a superposition of the Planck's distribution functions with slightly different temperatures. Even if this is the case, the energy scale of the particle creation emitted from the dynamically formed horizon is on the order of $T_{\mathrm{pc}}$.

We have simulated the expansion of the condensate by solving numerically (using the Crank-Nicolson scheme) the time-dependent GP equation. The initial groundstate wave function is obtained by solving the GP equation using the steepest descent method for an initial swave scattering length $a_{i}$ and the number of atoms $N$. We have computed $c_{s}$ and the radial velocity of the condensate via

$$
\begin{aligned}
& c_{s}=\sqrt{\left(\Psi^{*} \Psi\right) U_{0} / m}, \\
& v_{0}=\hbar\left[\Psi^{*} \partial_{r} \Psi-\left(\partial_{r} \Psi^{*}\right) \Psi\right] /\left(2 m i|\Psi|^{2}\right),
\end{aligned}
$$

and searched for parameter sets leading to $\left|v_{0}\right|>c_{s}$.

In the following, we assume that the condensate consists of $N=10^{5} \mathrm{Rb}$ atoms. (The values of atomic interaction given below are those in the case of $N=10^{5}$. If

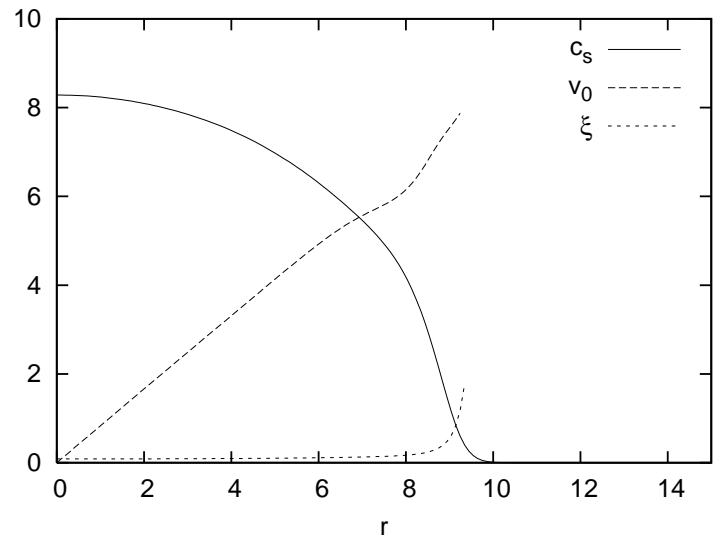

FIG. 2: Sound velocity $c_{s}$ (solid line) and the fluid velocity $v_{0}$ (dashed line) versus $r$ at $\tilde{t}=0.4$ in the case of $a_{i}=200 a_{0}$ and $a_{f}=5 a_{i}$ are shown in units of $\left(\hbar \omega_{h o} / m\right)^{1 / 2}$. The healing length $\xi$ (dotted line) is shown as well in units of $a_{h o}=\left(\hbar / m \omega_{h o}\right)^{1 / 2}$.

$N=10^{5} / n$ with an integer $n$, then $a_{i}$ and $a_{f}$ should be multiplied by $n$.) The initial atomic interaction is assumed to be $a_{i}=50 a_{0}, 200 a_{0}$ and $800 a_{0}$ where $a_{0}=0.53 \times 10^{-10} \mathrm{~m}$ is the Bohr radius. The following change of the atomic interaction has been simulated: $a_{f} / a_{i}=2,3,4,5,6,7,8,9,10,15,20,25,30$.

Just after $t=0$, the condensate begins to expand in the trapping potential and the expansion is accelerated for a while. At some time, say $t=t_{c}$, the expansion turns to be decelerated. Figure 1 shows $t_{c}$ as a function of $a_{f} / a_{i}$. It is seen that $t_{c}$ does not depend on the initial strength of the interaction. For $\tilde{t}:=\omega_{\text {ho }} t<\pi / 2$, the condensate continues to expand, and at $\tilde{t} \simeq \pi / 2$, the condensate starts to collapse. Therefore, we turn off the trapping potential at $\tilde{t}=\pi / 4$ and make the condensate expand freely in order to keep the horizon for a while.

As far as we have investigated, sonic horizon always appears in the sence of the surface where $v_{0}$ exceeds $c_{s}$. As an example of a sonic horizon, we show Fig. 2 which is the snapshot at $\tilde{t}=0.4$ in the case of $a_{i}=200 a_{0}$ and $a_{f}=5 a_{i}$. We see that, around $r=7 a_{h o}$, the fluid velocity exceeds the sound velocity, and the sonic horizon exists there. In this case, we find that, at $\tilde{t} \equiv \omega_{\text {ho }} t=0.11$, the horizon appears. Fig. 3 shows the time dependence of the radius of the horizon, say $r_{H}$, and the velocity gradient at the horizon $\left.\partial_{r}\left(v_{0}-c_{s}\right)\right|_{r_{H}}$.

If we keep the trapping potential for a long time, an oscillating behavior of the condensate is observed. The period of the oscillation is about $\pi$ in units of $\omega_{h o}^{-1}$ and we find $\omega_{\mathrm{BEC}} \simeq \omega_{h o}$, within a moderate change of the interaction. This oscillation is just like an oscillation of a droplet confined in a harmonic potential. Therefore, the condition (8) can be rewritten as

$$
\frac{c_{s}}{\xi \omega_{h o}} \gg 1
$$




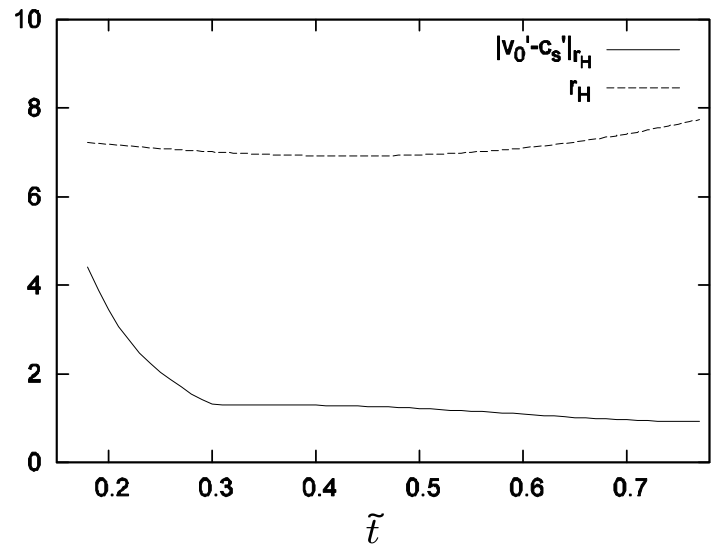

FIG. 3: Time dependence of $\partial_{r}\left(v_{0}-c_{s}\right)_{r_{H}}$ (solid line) in units of $\omega_{h o}$ and the position of the sonic horizon $r_{H}$ (dashed line) in units of $a_{h o}=\left(\hbar / m \omega_{h o}\right)^{1 / 2}$ in the case of $a_{i}=200 a_{0}$ and $a_{f}=5 a_{i}$.

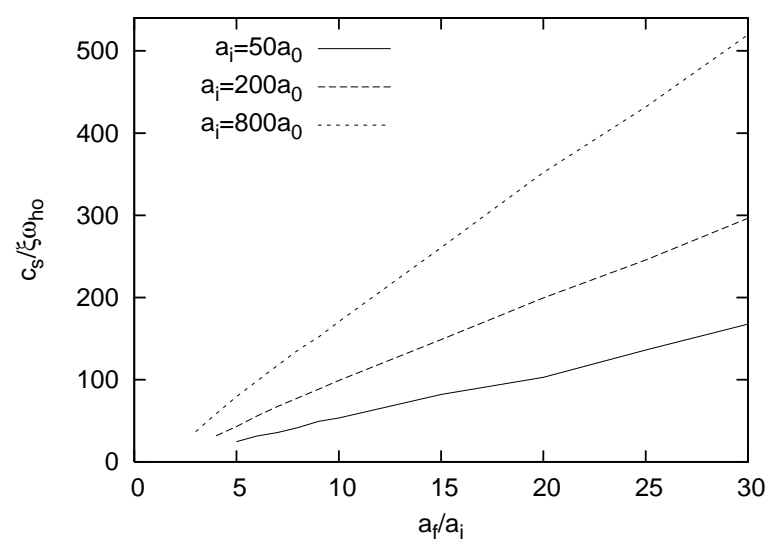

FIG. 4: $c_{s} / \xi \omega_{h o}$ as a function of $a_{f} / a_{i}$ is shown for each initial scattering length.

Now, we are interested in sonic horizon where (12) is satisfied. The intermediate region (7) exists if, for example, the following inequality is satisfied:

$$
\frac{c_{s}}{\xi \omega_{h o}} \geq 22.5
$$

For this choice of the lower bound, there exists a region of $\omega$ satisfying both conditions of $\omega \geq 10 \omega_{h o}$ and $\left(c_{s} / \omega \xi\right)^{2} \geq 5$. The condition (13) ensures hydrodynamic flow and quasi-static nature of the condensate. Fig. 4 shows $c_{s} / \xi \omega_{h o}$ as a function of $a_{f} / a_{i}$. We define horizon life time as the time interval during which the condition (13) continues to be satisfied at the horizon. The horizon life time is shown in Fig. 5. As far as we have investigated, the condensate flow satisfying (13) at sonic horizon appears only when $a_{f} \geq 5 a_{i}$ for $a_{i}=50 a_{0}, a_{f} \geq 4 a_{i}$ for $a_{i}=200 a_{0}$ and $a_{f} \geq 3 a_{i}$ for $a_{i}=800 a_{0}$.

The Hawking temperature at $t=t_{c}$ and $\tilde{t}=0.79$ (just

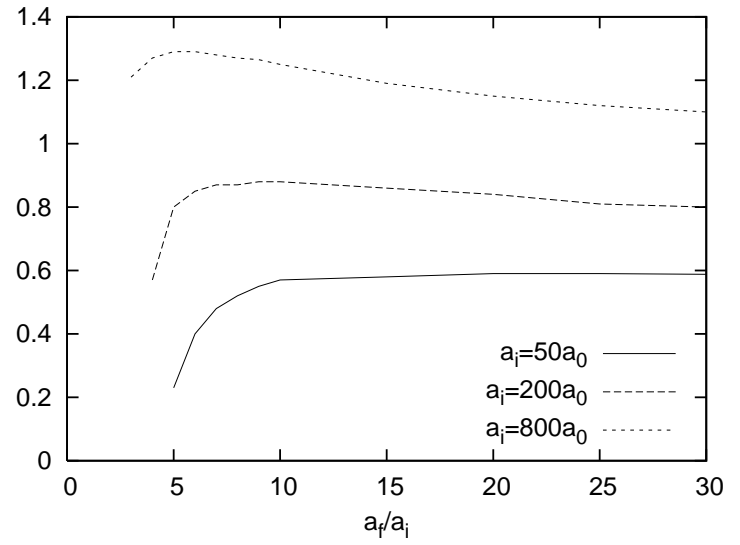

FIG. 5: Horizon life time as a function of $a_{f} / a_{i}$ is shown in units of $\omega_{h o}^{-1}$.

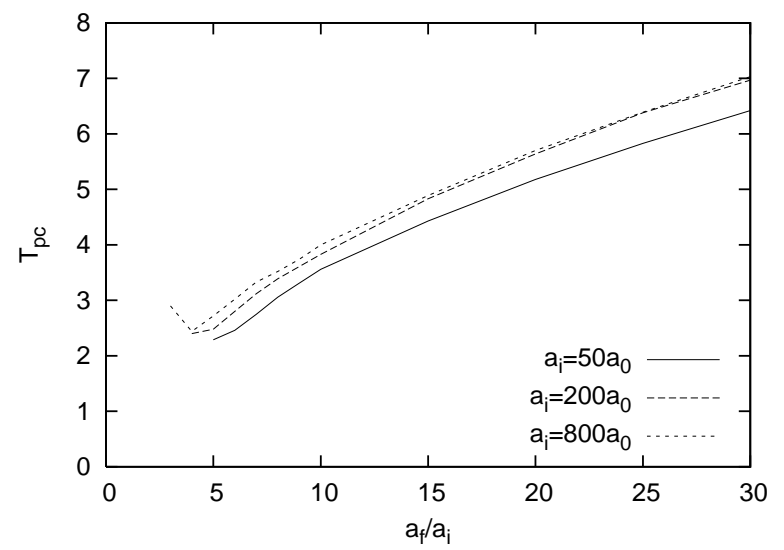

FIG. 6: Hawking temperature at $t=t_{c}$ in units of $n K$. In the evaluation, we assume $\omega_{h o}=1400 \mathrm{~Hz}$.

after turning off the trapping potential) are shown in Fig. 6 and Fig. 7 respectively. In the evaluation, we assume the frequency $\omega_{h o}=1400 \mathrm{~Hz}$. The Hawking temperature at $t=t_{c}$ depends on the ratio $a_{f} / a_{i}$ almost linearly. In contrast, for $a_{f} / a_{i} \geq 9$, the Hawking temperature at $\tilde{t}=0.79$ does not depend on the ratio so much. From the simulations, the temperature is expected to be a few $\mathrm{nK}$.

For this spherically symmetric trap, one may concern the three-body recombination loss of condensed atoms. Now, we check the effect of three-body losses for the given peak density. This effects may be taken into account by incorporating the imaginary term describing the inelastic process in the GP equation [22]

$$
i \hbar \partial_{t} \Psi=\left(-\frac{\hbar^{2}}{2 m} \nabla^{2}+V_{e x t}+U_{0}|\Psi|^{2}\right) \Psi-\frac{i \hbar}{2} K_{3}|\Psi|^{4} \Psi
$$

where $K_{3}$ denotes three-body recombination loss-rate coefficient. Then, the three-body loss is proportional to the 


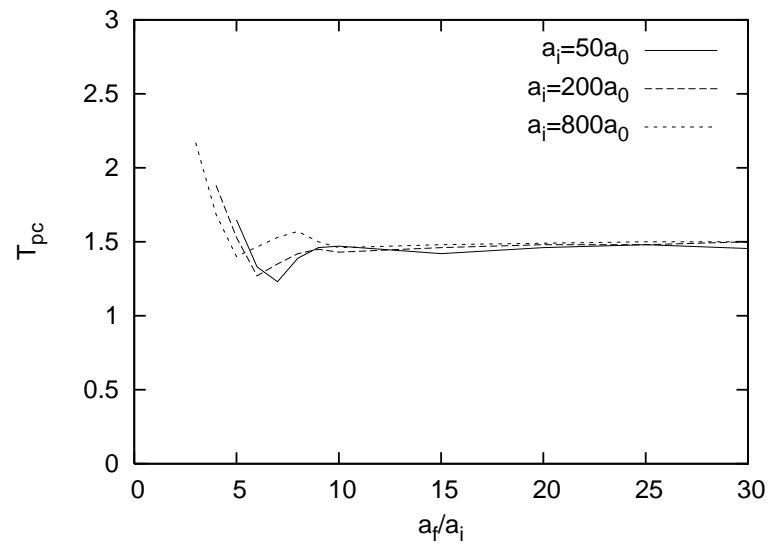

FIG. 7: Hawking temperature at $\tilde{t}=0.79$ in units of $n K$, when just after the trapping potential is turned off. In the evaluation, we assume $\omega_{h o}=1400 \mathrm{~Hz}$.

cube of the atomic density

$$
\frac{\partial}{\partial t} \int|\Psi|^{2} d^{3} r=-K_{3} \int|\Psi|^{6} d^{3} r
$$

which implies that the three-body loss rate is given by $R_{3} \equiv K_{3} \int|\Psi|^{6} d^{3} r / \int|\Psi|^{2} d^{3} r$. For the value of $K_{3}$, we assume $K_{3}=2 \times 10^{-28} \mathrm{~cm}^{6} / \mathrm{s}$, according to [23]. Of course, high atomic density causes many inelastic processes and gives high atomic loss rate. In our numerical simulation, the upper limit of the loss rate can be estimated by use of the peak density as $R_{3} \leq 3 \times 10 \mathrm{~s}^{-1}$, where the total atomic number was set to be $N=\int|\Psi|^{2} d^{3} \mathbf{r}=$ $10^{5}$. Then, the three-body loss can be ignored because we consider the time scale of $\leq 10 \mathrm{~ms}$.

In the above evaluation for Hawking temperature, horizon lifetime and $R_{3}$, we have assumed that the trapping frequency is $\omega_{h o}=1400 \mathrm{~Hz}$. Note that $\omega_{h o}$ is the energy scale of the system. Therefore, a large value of $\omega_{h o}$ is plausible to increase the characteristic temperature for the particle emission, though the time evolution process becomes rapid for large $\omega_{h o}$. If lower frequency is assumed, lower temperature, longer horizon lifetime and fewer three-body loss rate would be expected. As an example, Fig. 8 shows $\omega_{h o}$-dependence of the Hawking temperature and the horizon lifetime in the case of $a_{i}=200 a_{0}$ and $a_{f}=10 a_{i}$.

\section{BOGOLIUBOV SPECTRUM}

In the above numerical simulations, we assume there is no dynamical instability. Now, we check whether there is dynamical instability or not, within Gaussian approximation. For that purpose, we study the Bogoliubov-de Gennes equations: the second quantized field equations

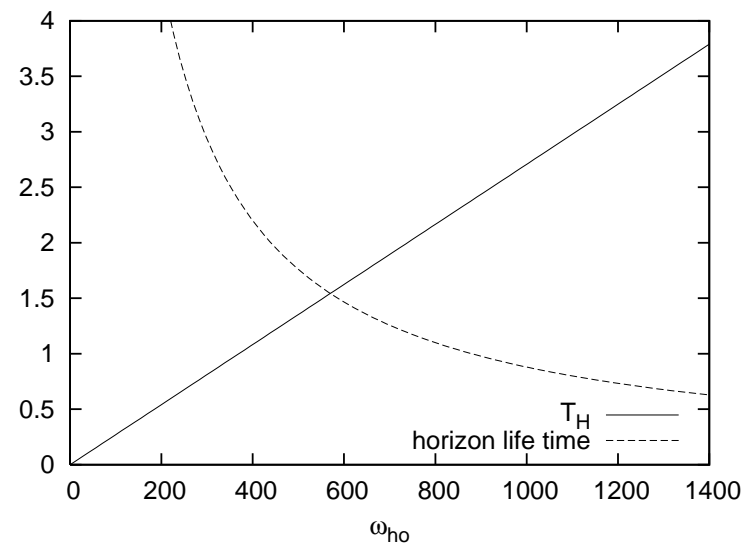

FIG. 8: $\omega_{h o}$-dependence of $T_{H}$ and horizon lifetime in the case of $a_{i}=200 a_{0}$ and $a_{f}=10 a_{i}$. The Hawking temperature is shown in units of nK and horizon lifetime is in units of $\omega_{h o}^{-1}$.

for the excitation fields $\delta \phi$ and $\overline{\delta \phi}$ are given by

$$
\begin{aligned}
i \hbar \partial_{t} \delta \phi & =\left(-\frac{\hbar^{2}}{2 m} \nabla^{2}+V_{\mathrm{ext}}+2 U_{0}|\Psi|^{2}\right) \delta \phi+U_{0} \Psi^{2} \overline{\delta \phi}, \\
-i \hbar \partial_{t} \overline{\delta \phi} & =\left(-\frac{\hbar^{2}}{2 m} \nabla^{2}+V_{\mathrm{ext}}+2 U_{0}|\Psi|^{2}\right) \overline{\delta \phi}+U_{0}(\bar{\Psi})^{2} \delta \phi .
\end{aligned}
$$

The excitation spectrum is computed by performing the Bogoliubov transformation:

$$
\begin{aligned}
& \delta \phi=\sum_{\alpha}\left[u_{\alpha}(\mathbf{r}) b_{\alpha} \mathrm{e}^{-i E_{\alpha} t / \hbar}-v_{\alpha}(\mathbf{r}) b_{\alpha}^{\dagger} \mathrm{e}^{i E_{\alpha} t / \hbar}\right] \\
& \overline{\delta \phi}=\sum_{\alpha}\left[u_{\alpha}^{*}(\mathbf{r}) b_{\alpha}^{\dagger} \mathrm{e}^{i E_{\alpha} t / \hbar}-v_{\alpha}^{*}(\mathbf{r}) b_{\alpha} \mathrm{e}^{-i E_{\alpha} t / \hbar}\right]
\end{aligned}
$$

The energy spectrum $E_{\alpha}$ is calculated by diagonalizing the skew symmetric matrix, which is carried out by using a routine in LAPACK. For the parameter values taken above, we find that all eigenvalues do not have the imaginary parts within numerical errors. Therefore, within Gaussian approximation, there is no dynamical instability. In addition, we find that there is no level crossing.

\section{SUMMARY}

To summarize, we have proposed an experiment to create a quasi-static sonic horizon using an expanding BEC. It has been shown that the dynamically formed quasi-static sonic horizon is in hydrodynamic regime as it should be to discuss analogy with curved spacetime in BEC. Under suitable choices of the interaction parameter and the confining potential, the characteristic temperature of the particle emission is expected to be a few $\mathrm{nK}$ for sufficiently strong confining potential. Large number of atoms or strong atomic interaction improves the quasi-static nature of the horizon. 
Of course, other effect such as cosmological particle creation can occur in this expanding BEC setup, as discussed in [14]-[21]. In this paper, we have focused on how to make dynamically formed quasi-static sonic horizon in the hydrodynamic regime of the condensate flow. In order to investigate cosmological particle creation effect and other excitations arising from depletion, we need a different numerical simulation scheme. The result will be reported in a future publication.

Furthermore, it is interesting to investigate numerically the behavior of negative frequency modes with positive norm which seem to be related to Hawking effect as was discussed in [26] 27]. This point shall be investigated in a future publication.

\section{Acknowledgments}

Y.K. thanks Hideki Ishihara, Ken-ichi Nakao, and Makoto Tsubota for useful discussions. The authors thank Michikazu Kobayashi and Takashi Uneyama for useful comments on numerical simulations. Y.K. was partially supported by the Yukawa memorial foundation. This work was also supported by the 21st Century COE "Center for Diversity and Universality in Physics" and "Constitution of wide-angle mathematical basis focused on knots" from the Ministry of Education, Culture, Sports, Science and Technology (MEXT) of Japan. The numerical calculations were carried out on Altix3700 BX2 at YITP in Kyoto University.

\section{APPENDIX A: PARTICLE CREATION PHENOMENON}

Here we focus on spherically symmetric quantum fluctuations by symmetry. At $t \leq 0$, the fluid velocity $v_{0}=0$, and the metric of the initial static effective spacetime is

$$
d s^{2} \propto-c_{s}^{2} d t^{2}+d r^{2}+r^{2} d \Omega_{S^{2}}^{2},
$$

where $d \Omega_{S^{2}}^{2}$ is the element of solid angle on the unit sphere $S^{2}$. After the increase of the interaction, the effective spacetime evolves dynamically as the BEC starts to expand. Then, the sonic horizon is formed as was shown by the above numerical simulation. If the effective spacetime is static, we can introduce a following time coordinate: $\tau=t+\int v_{0} d r /\left(c_{s}^{2}-v_{0}^{2}\right)$, and the effective spacetime metric becomes

$$
d s^{2} \propto-\left(c_{s}^{2}-v_{0}^{2}\right) d \tau^{2}+\frac{c_{s}^{2} d r^{2}}{c_{s}^{2}-v_{0}^{2}}+r^{2} d \Omega_{S^{2}}^{2} .
$$

From this expression, it is found that the horizon is located at the surface where the condition $c_{s}=\left|v_{0}\right|$ is satisfied. A new coordinate $v$ is introduced as $v \equiv \tau+r_{*}$ where $r_{*} \equiv \int c_{s} d r /\left(c_{s}^{2}-v_{0}^{2}\right)$, which is a coordinate characterizing ingoing light-like (null) rays in the effective spacetime.

We assume here that the initial state of the quantum field $\varphi$ is the vacuum state for the static observer in the initial effective spacetime. Under the time evolution of the effective spacetime caused by the expansion of the condensate, the creation and annihilation operators for the field $\varphi$ also evolve, and particle creation occurs.

Now we consider an observer who moves along his or her outgoing geodesic with proper time $\lambda$, crossing the horizon at $\lambda=0$. Hereafter, we term the observer geodesic observer. If we assume that the horizon is located at $r=r_{H}$, the proper time $\lambda$ is related to the coordinate $v$ there via $\lambda \approx-\lambda_{0} e^{-\frac{2 c_{H}}{\alpha} v}$, where $c_{H} \equiv c_{s}\left(r_{H}\right)$, $\left.\alpha \equiv 2 c_{H} \partial_{r}\left(v_{0}-c_{s}\right)\right|_{r=r_{H}}$ and $\lambda_{0}$ is a constant. The ingoing mode functions $\varphi_{\omega}=e^{-i \omega v}$ have $\lambda$-dependence near the horizon as

$$
\varphi_{\omega} \approx \exp \left(i \frac{2 c_{H} \omega}{\alpha} \ln (-\lambda)\right) .
$$

Initially, the state is the vacuum for the static observer and therefore the geodesic observer would see no excitation at short distance, because there will be no much higher positive frequency excitations than those determined by the time scale of the dynamical expansion of the BEC. If we ignore the short distance cut-off determined by the healing length, or equivalently, if the latter condition in Eq.(33) is ignored, this $\lambda$-dependence of the ingoing mode functions implies that the particle creation from the horizon into the inside of the condensate has thermal spectrum with the temperature given by (9). Furthermore, even if the short distance cut-off is taken into account, it is known that the result does not change in principle [25].

Therefore, the particle emission from horizon will occur in the case of expanding condensate, even where the subsonic region is inside of the horizon.
[1] S.W. Hawking, Nature 248, 30 (1974); S.W. Hawking, Commun. Math. Phys. 43, 199 (1975).

[2] Artificial Black Holes, edited by M. Novello, M. Visser, and G. Volovik (World Scientific, 2002).

[3] C. Barcelo, S. Liberati and M. Visser, Living Rev. Rel. 8, $12(2005)$

[4] W. G. Unruh, Phys. Rev. Lett. 46, 1351 (1981).
[5] M. H. Anderson, J. R. Ensher, M. R. Matthews, C. E. Wieman, and E.A. Cornell, Science 269, 198 (1995).

[6] K. B. Davis, M.-O. Mewes, M. R. Andrews, N. J. van Druten, D. S. Durfee, D. M. Kurn, and W. Ketterle, Phys. Rev. Lett. 75, 3969 (1995).

[7] L. J. Garay, J. R. Anglin, J. I. Cirac and P. Zoller, Phys. 
Rev. Lett. 85, 4643 (2000)

[8] L. J. Garay, J. R. Anglin, J. I. Cirac and P. Zoller, Phys. Rev. A 63, 023611 (2001)

[9] C. Barcelo, S. Liberati and M. Visser, Class. Quantum Grav. 18, 1137 (2001).

[10] S. Inouye, M. R. Andrews, J. Stenger, H.-J. Miesner, D. M. Stamper-Kurn, and W. Ketterle, Nature 392, 151 (1998); P. Courteille, R.S. Freeland, D.J. Heinzen, F.A. van Abeelen, and B.J. Verhaar, Phys. Rev. Lett. 81, 69 (1998); J.L. Roberts, N.R. Claussen, J.P. Burke, C.H. Greene, E.A. Cornell, and C. E. Wieman, Phys. Rev. Lett. 81, 5109 (1998).

[11] E. A. Donley, N. R. Claussen, S. L. Cornish, J. L. Roberts, E. A. Cornell, and C. E. Wieman, Nature 412, 295 (2001).

[12] E. A. Calzetta and B. L. Hu, Phys. Rev. A 68, 043625 (2003)

[13] E. A. Calzetta and B. L. Hu, cond-mat/0208569

[14] C. Barcelo, S. Liberati and M. Visser, Int.J.Mod. Phys. D 121641 (2003).

[15] C. Barcelo, S. Liberati and M. Visser, Phys. Rev. A 68 053613 (2003).
[16] P. O. Fedichev and U. R. Fischer, Phys. Rev. Lett. 91, 240407 (2003).

[17] P. O. Fedichev and U. R. Fischer, Phys. Rev. A. 69, 033602 (2004).

[18] P. O. Fedichev and U. R. Fischer, Phys. Rev. D 69, 064021 (2004).

[19] U. R. Fischer and R. Schützhold, Phys. Rev. A 70, 063615 (2004).

[20] J. E. Lidsey, Class. Quantum Grav. 21, 777 (2004).

[21] S. E. C. Weinfurtner, gr-qc/0404063

[22] Yu. Kagan, A.E. Muryshev, and G.V. Shlyapnikov, Phys. Rev. Lett. 81933 (1998).

[23] H. Saito and M. Ueda, Phys. Rev. A 65, 033624 (2002).

[24] For derivation details of the particle creation, see, for example, T. Jacobson, gr-qc/0308048.

[25] S. Corley and T. Jacobson, Phys. Rev. D 54, 1568 (1996).

[26] U. Leonhardt, T. Kiss and P. Öhberg, J. Opt. B 5 S 42 (2003).

[27] U. Leonhardt, T. Kiss and P. Öhberg, Phys. Rev. A 67, $033602(2003)$ 